\title{
Indicadores e fatores associados à sobrecarga em cuidadores informais de pacientes em radioterapia
} Indicators and factors associated with burden in informal caregivers of patients undergoing radiotherapy Indicadores y factores asociados a la sobrecarga de cuidadores informales de pacientes sometidos a radioterapia

Aliny de Oliveira Pedrosa ${ }^{1}$ (b) https://orcid.org/0000-0002-1396-9609 Daniel de Macêdo Rocha ${ }^{1}$ ib https:/orcid.org/0000-0003-1709-2143

Aline Costa de Oliveira ${ }^{1}$ @ https://orcid.org//0000-0003-1738-4808

Ana Maria Ribeiro dos Santos ${ }^{1}$ io https://orcid.org/0000-0002-5825-5335 Maria do Livramento Fortes Figueiredo ${ }^{1}$ io https://orcid.org/0000-0003-4938-2807 Lídya Tolstenko Nogueira ${ }^{1}$ io https://orcid.org/0000-0003-4918-6531

Como citar: Figueiredo ML, Nogueira LT. Indicadores e atores associados à sobrecarga em cuidadores informais de pacientes em radioterapia. Acta Paul Enferm. 2021;34:eAPE02924.

DOI http://dx.doi.org/10.37689/actaape/2021A002924

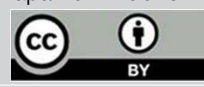

Descritores

Neoplasias; Radioterapia; Cuidadores; Carga de trabalho

Keywords

Neoplasms; Radiotherapy; Caregivers; Workload

Descriptores

Neoplasias; Radioterapia; Cuidadores; Carga de trabajo

Submetido 17 de Outubro de 2019 Aceito 2 de Dezembro de 2020

Autor correspondente

Aliny de Oliveira Pedrosa

E-mail: alinypedrosa08@hotmail.com

\section{Resumo}

Objetivo: Analisar os indicadores e fatores associados à sobrecarga em cuidadores informais de pacientes em radioterapia.

Métodos: Estudo transversal analítico, realizado com 209 cuidadores informais de pacientes em tratamento radioterápico. Para inclusão considerou-se ter idade superior a 18 anos, apresentar vínculo familiar, afetivo, relação de convivência ou coabitação e exercer cuidados há no mínimo 30 dias. Foram excluídos os cuidadores que durante a entrevista ausentaram-se para fins de acompanhamento do paciente, resultando no preenchimento incompleto dos instrumentos de coleta. Os dados foram coletados mediante formulários para caracterização dos cuidadores e pacientes, bem como avaliação da demanda de cuidados. A mensuração da sobrecarga foi realizada por meio do Questionário de Avaliação da Sobrecarga do Cuidador Informal. Utilizaram-se os testes t de Student para comparação dos escores médios de sobrecarga, ANOVA, MannWhitney, Kruskal-Wallis e Coeficiente de Correlação de Spearman para verificar relações entre as variáveis.

Resultados: Os escores médios demonstraram sobrecarga moderada, com maior impacto nos domínios "implicações na vida pessoal", "sobrecarga emocional" e "reações às exigências". Os fatores determinantes para o maior nível estiveram associados à demanda do cuidado, ao grau de parentesco e às condições clínicas e terapêuticas dos pacientes, dentre elas, idade, número de sessões de radioterapia prescritas, presença de comorbidades e sintomas físicos.

Conclusão: 0 cuidado informal na radioterapia gerou sobrecarga física, emocional e social nos cuidadores, acarretando implicações na vida pessoal e instabilidades emocionais. Aspectos relacionados à demanda de cuidados e às características dos pacientes foram determinantes para elevação dos escores globais.

\section{Abstract}

Objective: To analyze the indicators and factors associated with burden in informal caregivers of patients undergoing radiotherapy.

Methods: Analytical cross-sectional study conducted with 209 informal caregivers of patients undergoing radiotherapy. Age over 18 years old, having a family, affective, coexistence or cohabitation relationship and providing care for at least 30 days were considered as inclusion criteria. Caregivers who were absent during the interview for accompanying the patient were excluded, thereby resulting in incomplete completion of collection instruments. Data were collected with use of forms for characterization of caregivers and patients and assessment of the care demand. The burden was measured by means of the Informal Caregiver Burden Assessment Questionnaire. The Student's t-test was used to compare the mean burden scores, and the ANOVA, Mann-Whitney, Kruskal-Wallis and Spearman's correlation coefficient were used to assess the relationships between variables. 
Results: The mean scores showed moderate burden with a greater impact on the "implications for personal life", "emotional burden" and "reactions to demands" domains. The determining factors for the highest level were associated with the care demand, degree of kinship and the patients' clinical and therapeutic conditions, including age, number of prescribed radiotherapy sessions, presence of comorbidities and physical symptoms.

Conclusion: Informal care in radiotherapy generated physical, emotional and social burden on caregivers and had implications for personal life and emotional instabilities. Aspects related to the care demand and the characteristics of patients were decisive for the increase in global scores.

\section{Resumen}

Objetivo: Analizar los indicadores y factores asociados a la sobrecarga de cuidadores informales de pacientes sometidos a radioterapia.

Métodos: Estudio transversal analítico, realizado con 209 cuidadores informales de pacientes en tratamiento radioterápico. Para su inclusión, se consideraron las siguientes variables: tener edad superior a 18 años, presentar vínculo familiar, afectivo, relación de convivencia o cohabitación y ejercer cuidados durante 30 días como mínimo. Se excluyeron los cuidadores que, durante la entrevista, se ausentaron por motivos de atención al paciente, por lo que los instrumentos de recopilación quedaron incompletos. Los datos se recopilaron mediante formularios para la caracterización de los cuidadores y pacientes, así como también la evaluación de demandas de cuidados. La medición de la sobrecarga fue realizada mediante el Cuestionario de Evaluación de la Sobrecarga del Cuidador Informal. Se utilizó el test-T de Student para la comparación de la puntuación promedio de sobrecarga, ANOVA, Mann-Whitney, Kruskal-Wallis y Coeficiente de Correlación de Spearman para verificar relaciones entre las variables.

Resultados: Los puntajes promedio demostraron sobrecarga moderada, con mayor impacto en los dominios "consecuencias en la vida personal", "sobrecarga emocional" " "reacciones a las exigencias". Los factores determinantes del mayor nivel están relacionados con la demanda del cuidado, el nivel de parentesco y las condiciones clínicas y terapéuticas de los pacientes, entre ellas la edad, el número de sesiones de radioterapia prescriptas, la presencia de comorbilidades y los síntomas físicos.

Conclusión: El cuidado informal en la radioterapia generó sobrecarga física, emocional y social en los cuidadores, lo que conlleva consecuencias en la vida personal e inestabilidad emocional. Los aspectos relacionados con la demanda de cuidados y las características de los pacientes fueron determinantes para el aumento de la puntuación global.

\section{Introdução}

As modificaçóes na estrutura etária e no padrão de morbimortalidade é um fenômeno complexo de grande magnitude epidemiológica, econômica e social, que reflete diretamente nas condiçóes de saúde da população, resultando no aumento expressivo das doenças crônicas degenerativas, como o câncer, que apresenta elevada incidência e que constitui uma das causas de incapacidades e estado de dependência. ${ }^{(1)}$

Dentre os recursos terapêuticos estão: a quimioterapia, a intervenção cirúrgica e a radioterapia, que demandam apoio, reorganização e reestruturaçáo nos níveis familiar, social e de saúde, uma vez que o tratamento pode ocorrer em modalidade ambulatorial, necessitar de cuidados especializados em diferentes níveis de atenção e gerar sobrecarga para os cuidadores envolvidos. ${ }^{(2,3)}$

Apesar de evidenciadas as repercussóes do cuidado em diferentes modalidades terapêuticas, são incipientes as investigaçóes voltadas para radioterapia, que mesmo apresentando efetividade, pode ser acompanhada por eventos adversos imediatos e tardios. Tais eventos comprometem o grau de independência do paciente, levando à limitação funcional, intensificação de sintomas físicos, de reaçóes emocionais e dificuldades financeiras capazes de exigir conhecimento, disponibilidade de tempo e dedicação para o gerenciamento do cuidado domiciliar. ${ }^{(3-5)}$

Nessa perspectiva, membros familiares assumem responsabilidades como cuidadores, mesmo sem formação profissional ou preparo técnico, constituindo uma rede de suporte informal para gerenciar incapacidades, sintomatologias e eventos adversos. Assim, denomina-se cuidador informal aquela pessoa que presta assistência de forma não remunerada e que assume o cuidado por iniciativa ou denominação do grupo familiar, seja por relaçóes de parentesco, gênero, vínculo físico ou afetivo, representando, assim, uma das principais estratégias para enfrentamento da doença. ${ }^{(6)}$

A literatura considera que o cuidado informal ao paciente em tratamento radioterápico representa condição geradora de morbidade, estando diretamente associada à sobrecarga física, social, psicológica e financeira. ${ }^{(7)}$ Compreendida como reação biofísica multidimensional, a sobrecarga é resultante do desequilíbrio das demandas de cuidado em relação ao tempo pessoal, papéis sociais, estado físico, emocional e financeiro. Pode ser potencializada pelas deficiências no seu reconhecimento, tendo seu foco frequentemente ignorado ou negligenciado. ${ }^{(8,9)}$ 
Assim, a mensuração dos níveis de sobrecarga, bem como a identificação dos seus determinantes é amplamente referenciada, podendo ser realizada mediante instrumentos, questionários e escalas de medida desenvolvidas para determinar as limitaçóes impostas na vida do cuidador, conflitos conjugais, cansaço permanente, percepção de saúde piorada, além do adoecimento, exclusão social, comorbidades psicopatológicas, comprometimento do autocuidado e da qualidade de vida. ${ }^{(10)}$

Considerando a importância de compreender os preditores de sobrecarga, bem como a necessidade de estratégias assistenciais que fortaleçam o vínculo entre cuidador, equipe de saúde e paciente, definiu-se como objetivo do estudo analisar os indicadores e fatores associados à sobrecarga em cuidadores informais de pacientes em radioterapia.

\section{Métodos}

Estudo observacional, transversal e analítico, realizado no setor de radioterapia de uma instituição hospitalar referência para tratamento oncológico de Teresina, Piauí, entre janeiro e julho de 2018.

Dessa forma, a população de interesse foi constituída por cuidadores informais de pacientes em tratamento radioterápico. Para delineamento da amostra, foram consultados os indicadores epidemiológicos disponibilizados para acesso público no Portal-INCA, sendo identificado que as últimas estimativas para o local do estudo foi em 2014, correspondendo à 457 pacientes que realizaram radioterapia. ${ }^{(11)}$

Assim, foi utilizada a técnica de amostragem aleatória simples sem reposição, considerando intervalo de confiança de $95 \%$ e erro amostral de 5\%, o que resultou na seleção de 209 cuidadores, bem como dos seus respectivos pacientes.

Para inclusão foram considerados os seguintes critérios: ter idade igual ou superior a 18 anos, ser cuidador há no mínimo 30 dias e apresentar vínculo familiar, laços de afetividade, relação de convivência ou coabitaçáo com a pessoa em tratamento radioterápico. Foram excluídos os cuidadores que durante a entrevista ausentaram-se para fins de acompanhamento do paciente, resultando no preenchimento incompleto dos instrumentos de coleta.

A caracterização sociodemográfica e da demanda de cuidados, assim como a avaliação clínica e terapêutica dos pacientes foi realizada por meio de dois formulários, elaborados após revisão de literatura e submetidos à apreciação de especialistas para adequaçáo aos objetivos propostos.

Nessa perspectiva, foram investigadas variáveis relacionadas tanto ao cuidador quanto ao paciente. As relacionadas a cuidador foram: sexo, idade, estado civil, grau de parentesco, raça, escolaridade, procedência. A demanda de cuidados foi caracterizada pelas variáveis turno de trabalho, carga horaria de trabalho semanal e tempo destinado ao cuidado. As variáveis em relação aos pacientes foram: presença de comorbidades, localização do câncer, tempo de diagnóstico e de tratamento, número de sessóes de radioterapia prescritas e terapias adjuvantes.

Para a mensuração dos níveis de sobrecarga, utilizou-se o Questionário de Avaliação da Sobrecarga do Cuidador Informal (QASCI). Trata-se de uma ferramenta desenvolvida em Portugal, traduzida e validada para o Brasil. ${ }^{(10)}$

Tal ferramenta é constituída por 32 itens avaliados por uma escala ordinal de frequência que varia em cinco categorias de respostas ("Não/nunca", "Raramente", "Às vezes", "Quase sempre", "Sempre"), integrando, assim, sete dimensóes: "implicações na vida pessoal” (11 itens), "satisfação com o papel e com o familiar" (5 itens), "reaçôes às exigências" (5 itens), "sobrecarga emocional" (4 itens), "apoio familiar" (2 itens), "sobrecarga financeira" (2 itens) e "percepção dos mecanismos de eficácia e de controle" (3 itens). ${ }^{(10,12)}$

O escore total varia de 32 a 160 pontos, sendo calculado mediante a soma dos 32 itens, após a reversão das pontuaçóes correspondentes às três dimensões positivas ("percepção dos mecanismos de eficácia e de controle”, "apoio familiar e satisfação com o papel e com o familiar"). Assim, considera-se que valores mais altos correspondem a situaçôes com maior sobrecarga e que a média do escore total é aquela obtida no grupo de participantes. ${ }^{(10)}$ 
Desse modo, os dados foram coletados em ambiente reservado e disponibilizado pela instituição participante, após levantamento das pessoas que atendiam aos critérios de inclusão e seleção da amostra. Para tanto, realizou-se entrevista individualizada com cuidadores e pacientes, bem como análise de prontuários e registros clínicos. $\mathrm{O}$ tempo médio para avaliação foi de 30 minutos.

Os dados foram inseridos em dupla planilha no Software Microsoft Excel', para fins de validação, e transportados para o Statistical Package for Social Science (SPSS), visando à análise estatística com base nos princípios descritivos e inferenciais. A caracterização sociodemográfica dos cuidadores e clínicas dos pacientes foi expressa em medidas de tendência central (média e mediana), de variabilidade (desvio padrão) e em frequências absolutas e relativas.

$\mathrm{Na}$ análise inferencial, a avaliação da distribuição normal das variáveis contínuas foi realizada através do teste Kolmogorov-Smirnov. Para verificar associação entre os escores de sobrecarga com as variáveis sociodemográficas dos cuidadores, a demanda do cuidado e os aspectos demográficos, clínicos e terapêuticos dos pacientes aplicou-se os testes Kruskal-Wallis e Mann-Whitney em variáveis contínuas não-normais e os testes Análise de Variância (ANOVA) e t de Student para as normais. Utilizou-se o Coeficiente de Correlação de Spearman para verificar correlação entre os escores de sobrecarga e o QASCI global. O valor de $\mathrm{p}<$ 0,05 foi adotado como nível significativo para todos os testes.

Para interpretação da força de correlação, considerou-se a seguinte classificação: 0,00 a 0,20 - muito baixa; 0,21 a 0,39 - baixa; 0,40 a 0,69 - moderada; 0,70 a 0,89 - alta; 0,90 a 1,00 - muito alta; e igual a 1 - perfeita. ${ }^{(13)}$ Todas as análises foram realizadas ao nível de significância de 5\%, em que resultados com $\mathrm{p}$ valor igual ou inferior a 0,05 foram consideradas correlações positivas.

O estudo obteve aprovação e o parecer favorável à sua realização foi emitido pelo Comitê de Ética e Pesquisa da Universidade Federal do Piauí, sob processo de número 2.379.697. A participação foi condicionada à assinatura do Termo de Consentimento Livre e Esclarecido.

\section{Resultados}

Na tabela 1, está apresentada a caracterização dos participantes, assim como a descrição da demanda de cuidado. Predominara cuidadores do sexo feminino ( $\mathrm{n}=165,78,9 \%$ ), com idade média de 39,7 anos, casados ( $\mathrm{n}=114,54,5 \%)$, com ensino médio completo $(n=98,46,9 \%)$, desempregados $(n=105$, $50,2 \%)$ e procedentes de Teresina $(n=84,40,2 \%)$. Em relação ao cuidado informal, foram evidenciadas relaçóes fraternas de filhos $(n=86,41,1 \%)$, que exerciam essa função há mais de seis meses $(n=62$, $44,0 \%$ ), com período superior a 12 horas por dia (n $=129,61,7 \%)$.

Os pacientes assistidos pelo cuidador apresentavam as seguintes características: idade média de $58,5$ anos, sexo feminino ( $\mathrm{n}=128,61,2 \%)$, com câncer localizado na região da cabeça e pescoço (n $=46,22,0 \%$ ), diagnosticados há mais de seis meses ( $\mathrm{n}=94,44,9 \%$ ), em radioterapia e quimioterapia concomitantes ( $\mathrm{n}=80,38,3 \%)$, com 21 a 30 sessôes prescritas $(\mathrm{n}=76,36,4 \%)$ e relato de histórico familiar da doença $(n=107,51,2 \%)$.

Ainda, os pacientes referiram a dor foi como o principal sintoma apresentado durante o tratamento $(\mathrm{n}=120,57,4 \%)$ e reportaram as alteraçóes cutâneas $(\mathrm{n}=57,27,3 \%)$ como evento adverso à radioterapia, que envolveram a necessidade de trocas diárias de curativo $(\mathrm{n}=25,12,0 \%)$. Destacaram-se como comorbidades associadas: hipertensão arterial sistêmica ( $\mathrm{n}=79,37,8 \%)$, diabetes mellitus ( $\mathrm{n}=$ $50,23,9 \%)$ e, como hábitos, sedentarismo ( $\mathrm{n}=77$, $36,8 \%)$, tabagismo $(\mathrm{n}=70,33,5 \%)$ e etilismo $(\mathrm{n}=$ 47, 22,5\%).

Os resultados expressos na tabela 2 demonstram os escores médios de sobrecarga, assim como a correlação entre as sete dimensôes e o QASCI total. Observa-se que os maiores comprometimentos se concentraram nos domínios "implicaçôes na vida pessoal" $(31,50 \pm 13,57)$, "sobrecarga emocional" (11,0 $\pm 5,1)$ e "reaçóes às exigências" (11,0 \pm $6,2)$, sendo considerados os principais indicadores dos níveis de sobrecarga relacionada ao cuidado $(\mathrm{p}<0,001)$.

A tabela 3 apresenta a comparação entre as características sociodemográficas dos cuidadores 
Tabela 1. Variáveis de caracterização dos participantes e da demanda de cuidados

\begin{tabular}{|c|c|c|c|c|}
\hline \multirow{2}{*}{ Variável } & \multicolumn{2}{|c|}{ Cuidador } & \multicolumn{2}{|c|}{ Paciente } \\
\hline & $n(\%)$ & Média(DP) & $\mathrm{n}(\%)$ & Média(DP) \\
\hline \multicolumn{5}{|l|}{ Sexo } \\
\hline Masculino & $44(21,1)$ & & $81(38,8)$ & \\
\hline Feminino & $165(78,9)$ & & $128(61,2)$ & \\
\hline Idade & & $39,7(13,9)$ & & $58,5(15,2)$ \\
\hline \multicolumn{5}{|l|}{ Estado civil } \\
\hline Casado & $114(54,5)$ & & & \\
\hline Solteiro & $68(32,5)$ & & & \\
\hline Separado & $18(8,6)$ & & & \\
\hline Viúvo & $9(4,4)$ & & & \\
\hline \multicolumn{5}{|l|}{ Escolaridade } \\
\hline Analfabeto & $4(1,9)$ & & & \\
\hline Ensino fundamental incompleto & $33(15,8)$ & & & \\
\hline Ensino fundamental completo & $49(23,5)$ & & & \\
\hline Ensino médio completo & $98(46,9)$ & & & \\
\hline Ensino superior completo & $25(11,9)$ & & & \\
\hline \multicolumn{5}{|l|}{ Situação laboral } \\
\hline Trabalho formal & $48(23,0)$ & & & \\
\hline Trabalho informal & $43(20,6)$ & & & \\
\hline Desempregado & $105(50,2)$ & & & \\
\hline Aposentado & $13(6,2)$ & & & \\
\hline \multicolumn{5}{|l|}{ Grau de parentesco } \\
\hline Irmão & $36(17,2)$ & & & \\
\hline Filho & $86(41,1)$ & & & \\
\hline Pai ou mãe & $16(7,6)$ & & & \\
\hline Cônjuge & $34(16,3)$ & & & \\
\hline Neto (a) & $5(2,4)$ & & & \\
\hline Sobrinho (a) & $29(13,9)$ & & & \\
\hline Tio (a) & $1(0,5)$ & & & \\
\hline Amigo (a) & $1(0,5)$ & & & \\
\hline Cunhado (a) & $1(0,5)$ & & & \\
\hline \multicolumn{5}{|l|}{ Turno de trabalho } \\
\hline Manhã & $28(13,4)$ & & & \\
\hline Tarde & $4(1,9)$ & & & \\
\hline Noite & $1(0,5)$ & & & \\
\hline Dois turnos & $50(23,9)$ & & & \\
\hline Três turnos & $8(3,8)$ & & & \\
\hline Não se aplica & $118(56,5)$ & & & \\
\hline \multicolumn{5}{|l|}{ Carga horária de trabalho semanal } \\
\hline Até 20 horas semanais & $33(15,8)$ & & & \\
\hline Até 30 horas semanais & $34(16,3)$ & & & \\
\hline Até 40 horas semanais & $20(9,6)$ & & & \\
\hline Mais de 40 horas semanais & $4(1,9)$ & & & \\
\hline Não se aplica & $118(56,4)$ & & & \\
\hline \multicolumn{5}{|l|}{ Tempo destinado ao cuidado } \\
\hline Até 4 horas por dia & $34(16,3)$ & & & \\
\hline Até 8 horas por dia & $34(16,3)$ & & & \\
\hline Até 12 horas por dia & $12(5,7)$ & & & \\
\hline Mais de 12 horas por dia & $129(61,7)$ & & & \\
\hline \multicolumn{5}{|l|}{ Tempo de cuidador } \\
\hline De 1 a 3 meses & $61(29,2)$ & & & \\
\hline De 4 a 6 meses & $56(26,8)$ & & & \\
\hline Superior a 6 meses & $92(44,0)$ & & & \\
\hline \multicolumn{5}{|l|}{ Localização do câncer" } \\
\hline Cabeça e pescoço & & & $46(22,0)$ & \\
\hline Mama & & & $34(16,3)$ & \\
\hline Pulmão & & & $12(5,7)$ & \\
\hline Próstata & & & $38(18,2)$ & \\
\hline
\end{tabular}

\begin{tabular}{|c|c|c|c|c|}
\hline \multirow{2}{*}{ Variável } & \multicolumn{2}{|c|}{ Cuidador } & \multicolumn{2}{|c|}{ Paciente } \\
\hline & $\mathrm{n}(\%)$ & Média(DP) & $\mathrm{n}(\%)$ & Média(DP) \\
\hline Pâncreas & & & $1(0,48)$ & \\
\hline Colo do útero & & & $45(21,5)$ & \\
\hline Colorretal & & & $14(6,7)$ & \\
\hline Pele & & & $19(9,1)$ & \\
\hline \multicolumn{5}{|l|}{ Tempo de diagnóstico } \\
\hline Até 3 meses & & & $57(27,3)$ & \\
\hline 4 a 6 meses & & & $58(27,8)$ & \\
\hline Mais de 6 meses & & & $94(44,9)$ & \\
\hline \multicolumn{5}{|l|}{ Tratamento realizado ${ }^{\#}$} \\
\hline Radioterapia & & & $35(16,7)$ & \\
\hline Radioterapia e quimioterapia & & & $80(38,3)$ & \\
\hline Radioterapia e cirurgia & & & $52(24,9)$ & \\
\hline $\begin{array}{l}\text { Radioterapia, cirurgia e } \\
\text { quimioterapia }\end{array}$ & & & $42(20,1)$ & \\
\hline Presença de lesões de pele* & & & $57(27,3)$ & \\
\hline \multicolumn{5}{|l|}{ Comorbidades e fatores de risco ${ }^{\#}$} \\
\hline Diabético* & & & $50(23,9)$ & \\
\hline Hipertenso* & & & $79(37,8)$ & \\
\hline Tabagista* & & & $70(33,5)$ & \\
\hline Etilista $^{*}$ & & & $47(22,5)$ & \\
\hline Sedentário* & & & $77(36,8)$ & \\
\hline Histórico familiar do paciente ${ }^{*}$ & & & $107(51,2)$ & \\
\hline \multicolumn{5}{|l|}{ Sintomatologias } \\
\hline Dor* & & & $120(57,4)$ & \\
\hline Náuseas* & & & $48(23,0)$ & \\
\hline Vômitos* & & & $32(15,3)$ & \\
\hline Fadiga* & & & $98(46,9)$ & \\
\hline Ansiedade ${ }^{\star}$ & & & $32(15,3)$ & \\
\hline Outros sintomas* & & & $15(7,2)$ & \\
\hline
\end{tabular}

DP - Desvio Padrão; " A soma das frequências é superior a 100\% uma vez que participante pode ter apresentado mais de uma condição associada; ${ }^{\star}$ Consideradas apenas as respostas afirmativas.

Tabela 2. Escores médios de sobrecarga e correlação entre os domínios e escore global do QASCI

\begin{tabular}{lcccc}
\hline Dimensões & Média $\pm \mathrm{DP}$ & $\begin{array}{c}\rho \text { de } \\
\text { Spearman }\end{array}$ & p-value \\
\hline QASCl & $74,6 \pm 25,5$ & & \\
Sobrecarga emocional & $11,0 \pm 5,1$ & 0,737 & $<0,001$ \\
Implicações na vida pessoal & $31,5 \pm 13,5$ & 0,906 & $<0,001$ \\
Sobrecarga financeira & $5,7 \pm 3,1$ & 0,671 & $<0,001$ \\
Reações às exigências & $11,0 \pm 6,2$ & 0,800 & $<0,001$ \\
Percepção dos mecanismos de eficácia e controle & $5,58 \pm 2,8$ & 0,183 & 0,008 \\
Apoio familiar & $3,3 \pm 2,2$ & 0,305 & $<0,001$ \\
Satisfação com o papel e com o familiar & $6,4 \pm 2,9$ & 0,358 & $<0,001$ \\
\hline
\end{tabular}

QASCI - Questionário de Avaliação da Sobrecarga do Cuidador Informal; 0 -value foi obtido pelo teste de correlação $\rho$ de Spearman

e a demanda de cuidados com os escores globais. Dentre as associaçóes, constatou-se que as variáveis idade, renda familiar, situação laboral, carga horária semanal e turno de trabalho, grau de parentesco, procedência e tempo destinado ao cuidado contribuíram diretamente para maior sobrecarga $(\mathrm{p}<0,05)$, especificamente nos domínios "implicaçôes na vida pessoal", "sobrecarga emocional" e "sobrecarga financeira”. 
Tabela 3. Relação entre os escores de sobrecarga com as variáveis sociodemográficas dos cuidadores e a demanda de cuidados

\begin{tabular}{|c|c|c|c|c|c|c|c|c|}
\hline \multirow[t]{2}{*}{ Aspectos sociodemográficos dos cuidadores } & $\begin{array}{l}\text { QASCI } \\
\text { Total }\end{array}$ & SE & IVP & SF & RE & PMEC & AF & SPF \\
\hline & \multicolumn{8}{|c|}{$p$-value } \\
\hline Sexo & $0,591^{2}$ & $0,450^{1}$ & $0,984^{1}$ & $0,370^{1}$ & $0,402^{1}$ & $0,666^{1}$ & $0,511^{1}$ & $0,164^{1}$ \\
\hline Idade & $0,047^{5}$ & $0,253^{5}$ & $0,019^{5}$ & $0,357^{5}$ & $0,028^{5}$ & $0,002^{5}$ & $0,053^{5}$ & $0,325^{5}$ \\
\hline Estado civil & $0,231^{3}$ & $0,133^{3}$ & $0,198^{3}$ & $0,020^{3}$ & $0,084^{3}$ & $0,465^{3}$ & $0,182^{3}$ & $0,113^{3}$ \\
\hline Escolaridade & $0,404^{3}$ & $0,990^{3}$ & $0,137^{3}$ & $0,170^{3}$ & $0,432^{3}$ & $0,624^{3}$ & $0,105^{3}$ & $0,478^{3}$ \\
\hline Raça ou cor & $0,223^{3}$ & $0,400^{3}$ & $0,133^{3}$ & $0,020^{3}$ & $0,165^{3}$ & $0,727^{3}$ & $0,648^{3}$ & $0,261^{3}$ \\
\hline Situação laboral & $0,016^{3}$ & $0,417^{3}$ & $0,004^{3}$ & $0,259^{3}$ & $0,031^{3}$ & $0,309^{3}$ & $0,833^{3}$ & $0,102^{3}$ \\
\hline Turno de trabalho & $0,002^{3}$ & $0,100^{3}$ & $0,005^{3}$ & $0,021^{3}$ & $0,035^{3}$ & $0,095^{3}$ & $0,373^{3}$ & $0,169^{3}$ \\
\hline Carga horária de trabalho semanal & $0,001^{3}$ & $0,030^{3}$ & $0,001^{3}$ & $0,045^{3}$ & $0,001^{3}$ & $0,698^{3}$ & $0,696^{3}$ & $0,152^{3}$ \\
\hline Renda familiar & $0,006^{3}$ & $0,010^{3}$ & $0,122^{3}$ & $0,005^{3}$ & $0,004^{3}$ & $0,745^{3}$ & $0,048^{3}$ & $0,513^{3}$ \\
\hline Procedência & $0,004^{4}$ & $<0,001^{3}$ & $0,055^{3}$ & $0,432^{3}$ & $0,004^{3}$ & $0,388^{3}$ & $0,836^{3}$ & $0,366^{3}$ \\
\hline Tempo destinado ao cuidado & $<0,001^{3}$ & $<0,001^{3}$ & $<0,001^{3}$ & $0,002^{3}$ & $<0,001^{3}$ & $0,047^{3}$ & $0,041^{3}$ & $0,003^{3}$ \\
\hline Grau de parentesco & $<0,001^{3}$ & $0,003^{3}$ & $0,001^{3}$ & $0,148^{3}$ & $<0,001^{3}$ & $0,153^{3}$ & $0,676^{3}$ & $0,247^{3}$ \\
\hline Tempo de cuidador & $0,001^{4}$ & $0,160^{3}$ & $<0,001^{3}$ & $0,082^{3}$ & $<0,001^{3}$ & $0,126^{3}$ & $0,295^{3}$ & $0,015^{3}$ \\
\hline
\end{tabular}

SE - Sobrecarga Emocional; IVP - Implicações na Vida Pessoal; SF - Sobrecarga Financeira; RE - Reações às Exigências; PMEC - Percepção dos Mecanismos de Eficácia e Controle; AF - Apoio Familiar; SPF - Satisfação com o Papel e com o Familiar; 1 - Mann-Whitney, 2 - t de Student, 3 - Kruskall-Wallis; 4 - ANOVA; 5 - Coeficiente de Correlação de Spearmann

Tabela 4. Associação entre os escores de sobrecarga e os aspectos demográficos e clínicos dos pacientes

\begin{tabular}{|c|c|c|c|c|c|c|c|c|}
\hline \multirow{2}{*}{$\begin{array}{l}\text { Aspectos demográficos e clínicos do paciente } \\
\text { oncológico }\end{array}$} & $\begin{array}{l}\text { QASCl } \\
\text { Total }\end{array}$ & SE & IVP & SF & RE & PMEC & AF & SPF \\
\hline & \multicolumn{8}{|c|}{$p$-value } \\
\hline Sexo & $0,087^{1}$ & $0,225^{1}$ & $0,038^{1}$ & $0,809^{1}$ & $0,495^{1}$ & $0,418^{1}$ & $0,307^{1}$ & $0,318^{1}$ \\
\hline Idade & $0,047^{5}$ & $0,253^{5}$ & $0,019^{5}$ & $0,357^{5}$ & $0,028^{5}$ & $0,002^{5}$ & $0,053^{5}$ & $0,325^{5}$ \\
\hline Tempo de diagnóstico & $<0,001^{4}$ & $0,253^{3}$ & $0,002^{3}$ & $0,039^{3}$ & $0,002^{3}$ & $0,125^{3}$ & $0,026^{3}$ & $0,004^{3}$ \\
\hline Tratamento realizado & $0,057^{4}$ & $0,012^{3}$ & $0,025^{3}$ & $0,355^{3}$ & $0,647^{3}$ & $0,435^{3}$ & $0,764^{3}$ & $0,853^{3}$ \\
\hline Número de sessões prescritas & $<0,001^{3}$ & $0,002^{3}$ & $<0,001^{3}$ & $<0,001^{3}$ & $<0,001^{3}$ & $0,620^{3}$ & $0,537^{3}$ & $0,345^{3}$ \\
\hline Presença de lesões de pele & $0,991^{1}$ & $0,045^{1}$ & $0,747^{1}$ & $0,991^{1}$ & $0,762^{1}$ & $0,387^{1}$ & $0,657^{1}$ & $0,368^{1}$ \\
\hline Diabético & $<0,001^{2}$ & $0,002^{1}$ & $<0,001^{1}$ & $0,002^{1}$ & $<0,001^{1}$ & $0,954^{1}$ & $0,312^{1}$ & $0,005^{1}$ \\
\hline Hipertenso & $0,001^{1}$ & $0,002^{1}$ & $<0,001^{1}$ & $0,291^{1}$ & $0,008^{1}$ & $0,926^{1}$ & $0,750^{1}$ & $0,525^{1}$ \\
\hline Tabagista & $0,172^{2}$ & $0,141^{1}$ & $0,136^{1}$ & $0,785^{1}$ & $0,094^{1}$ & $0,181^{1}$ & $0,114^{1}$ & $0,662^{1}$ \\
\hline Etilista & $0,002^{1}$ & $0,034^{1}$ & $0,003^{1}$ & $0,056^{1}$ & $0,005^{1}$ & $0,577^{1}$ & $0,132^{1}$ & $0,553^{1}$ \\
\hline Sedentário & $0,008^{1}$ & $0,013^{1}$ & $0,035^{1}$ & $0,275^{1}$ & $0,098^{1}$ & $0,100^{1}$ & $0,186^{1}$ & $0,394^{1}$ \\
\hline Presença de dor & $<0,001^{2}$ & $0,176^{1}$ & $0,005^{1}$ & $0,005^{1}$ & $0,007^{1}$ & $0,160^{1}$ & $0,015^{1}$ & $0,033^{1}$ \\
\hline Vômitos & $0,031^{1}$ & $0,070^{1}$ & $0,095^{1}$ & $0,001^{1}$ & $0,315^{1}$ & $0,122^{1}$ & $0,696^{1}$ & $0,125^{1}$ \\
\hline Fadiga & $<0,001^{2}$ & $0,021^{1}$ & $<0,001^{1}$ & $0,189^{1}$ & $<0,001^{1}$ & $0,712^{1}$ & $0,962^{1}$ & $0,812^{1}$ \\
\hline Ansiedade & $0,014^{1}$ & $0,116^{1}$ & $0,022^{1}$ & $0,524^{1}$ & $0,047^{1}$ & $0,226^{1}$ & $0,174^{1}$ & $0,084^{1}$ \\
\hline Metástase & $0,720^{1}$ & $0,885^{1}$ & $0,270^{1}$ & $0,911^{1}$ & $0,389^{1}$ & $0,976^{1}$ & $0,027^{1}$ & $0,812^{1}$ \\
\hline
\end{tabular}

SE - Sobrecarga Emocional; IVP - Implicações na Vida Pessoal; SF - Sobrecarga Financeira; RE - Reações às Exigências; PMEC - Percepção dos Mecanismos de Eficácia e Controle; AF - Apoio Familiar; SPF - Satisfação com 0 Papel e com o Familiar; 1 - Mann-Whitney, 2 - t de Student, 3 - Kruskall-Wallis; 4 - ANOVA; 5 - Coeficiente de Correlação de Spearmann

A tabela 4 evidencia que variáveis clínicas e terapêuticas dos pacientes como tempo de diagnóstico, número de sessóes de radioterapia, ser hipertenso ou diabético e apresentar dor ou vômito estiveram associadas à elevação dos escores globais, com maior comprometimento nos domínios "sobrecarga emocional", "sobrecarga financeira", "implicaçóes na vida pessoal", "reaçóes às exigências" e "satisfação com o papel e com o familiar".

\section{Discussão}

O cuidado informal, conforme evidenciado neste estudo, constituiu um fenômeno complexo, estan- do associado a diferentes fatores que envolveram tanto a demanda de cuidados quanto os aspectos clínicos e terapêuticos dos pacientes que contribuíram para elevaçáo dos escores globais de sobrecarga e para estabelecer os impactos no bem-estar físico, mental e social, bem como no funcionamento pessoal, familiar e financeiro.

A sobrecarga vivenciada pelo cuidador representa indicador de impactos negativos e decorre do despreparo para assumir todas as responsabilidades que lhes são postas, sem conhecimento, apoio ou suporte para gerenciar situaçóes inesperadas ou condiçóes adversas. Pode ainda estar associada a contextos de vulnerabilidades, em que múltiplos fatores levam ao desgaste, acarretando mudanças no estilo de vida, 
manifestações físicas e reaçóes emocionais como incapacidades, limitaçóes funcionais e comorbidades psicopatológicas. ${ }^{(14-18)}$

Foram considerados como indicadores de sobrecarga as implicaçôes na vida pessoal e os comprometimentos emocionais, sendo determinantes para elevação dos escores globais. Dentre os fatores associados, verificou-se que aspectos relacionados tanto à demanda de cuidados, quanto às características clínicas e terapêuticas dos pacientes, tais como tempo destinado ao cuidado, grau de parentesco, presença de comorbidades e de sintomas físicos influenciaram no nível de sobrecarga referida pelos cuidadores.

Nesse contexto, comprometimentos no domínio "implicações na vida pessoal" prevaleceram, evidenciando pior percepção sobre a condição de saúde, maior esforço físico, negligência do autocuidado, abandono das atividades laborais e interrupção de planos futuros, refletindo negativamente na qualidade do cuidado prestado, favorecendo o aumento das incapacidades e dificultando o processo de reabilitação. ${ }^{(10)}$

Assim como em outros estudos, os domínios "sobrecarga emocional" e "reaçóes às exigências" também exerceram influências sobre os escores globais, indicando maior sobrecarga do cuidador. ${ }^{(15,19,20)}$ Os impactos emocionais referidos pelos cuidadores correspondem ao conjunto de emoçóes e sentimentos negativos, sintomas de ansiedade e depressão, sensação de cansaço, esgotamento e desesperança, desejo de fuga e conflitos internos, na maioria das vezes, potencializados pelo despreparo em cuidar. ${ }^{(14,10)}$

A "sobrecarga financeira" vivenciada pelos participantes pode ser reflexo da realidade brasileira no segmento social, educacional e econômico, em que estados de dependência limitam o acesso à educação e geram instabilidades laborais e familiares.

Essas dificuldades podem ser justificadas pelo abandono das atividades laborais para priorização das necessidades do paciente, pelo contexto neoliberal contemporâneo que exige a multifuncionalidade dos trabalhadores e pelo fato de a ausência de centros especializados gerar mudanças na rotina de vida, causando o distanciamento familiar e requerendo investimentos em hospedagens, locomoçáo, consultas, procedimentos e recursos diagnósticos e terapêuticos. ${ }^{(21,22)}$

Os menores escores de sobrecarga se concentraram na dimensão "apoio familiar", considerada principal estratégia para enfrentamento do câncer e dos eventos adversos decorrentes do tratamento adotado. A formação e fortalecimento de redes de suporte social frente às condiçóes crônicas e incapacitantes minimizam as manifestaçôes clínicas e as repercussóes do cuidado, como sintomas físicos, depressivos e ansiosos, uma vez que a responsabilidade é compartilhada entre diferentes membros familiares. ${ }^{(17,23)}$

Em relação aos fatores associados ao nível de sobrecarga referida, verificou-se que a variável grau de parentesco apresentou significância, constatando maior demanda do cuidado dispensado pelos filhos, papel determinado historicamente por valores sociais e culturais. A relação afetiva entre paciente e cuidador pode contribuir para sobrecarga, pelo acúmulo de papéis, quando encarada como obrigação. Entretanto, na maioria das vezes a manutenção desse vínculo pode favorecer a inserção e a adaptação à rotina de cuidados, reduzindo os escores de sobrecarga e os sentimentos negativos. ${ }^{(24)}$

Associação positiva também foi evidenciada entre o estado civil e domínio "sobrecarga financeira". O predomínio de pessoas casadas, referidas como provedoras do lar, contribuiu para sobrecarga, tendo em vista a necessidade de reduzir a carga horária ou de abandonar as atividades laborais para realizar o cuidado familiar e buscar recursos para financiar os custos com o tratamento. ${ }^{(25-27)}$

O desemprego decorrente, na maioria dos casos, da necessidade de afastamento laboral para dedicação ao cuidado informal foi considerado importante indicador de vulnerabilidade e determinante para as dimensôes "implicaçôes na vida pessoal" e "reações às exigências". Esse resultado pode ser justificado pela condição crônica da doença que demanda tempo do cuidador, limitando a ascensão social e econômica, além de dificultar o acesso aos serviços de saúde, às informaçóes e aos recursos terapêuticos. ${ }^{(24,28)}$

Ao que concerne à demanda do cuidado informal, ser cuidador há mais de seis meses, bem como dedicar mais de 12 horas por dia para gerenciar as 
necessidades do paciente são aspectos que favorecem maior sobrecarga física, emocional e social. Isso pode ser resultante das limitaçóes na vida pessoal e do desenvolvimento de reações emocionais, visto que durante a radioterapia cuidados específicos podem surgir, como a troca diária de curativos em dermatites induzidas pela radiação que acarretam elevado grau de destruição tecidual. ${ }^{(29)}$

Todavia, um estudo mostra que o tempo prolongado em que o cuidador se dedica a assistir o familiar doente e gerenciar suas necessidades, pode favorecer a adaptaçáo emocional e o estabelecimento de estratégias que promovam o fortalecimento de vínculo entre familiares, paciente e equipe de saúde. ${ }^{(30)}$

Variáveis relacionadas às características clínicas e terapêuticas dos pacientes como tempo de diagnóstico, número de sessôes de radioterapia, apresentar comorbidades ou sintomatologias físicas também estiveram associadas aos escores médios de sobrecarga. Nessa perspectiva, a presença de complicaçóes clínicas, comprometimentos funcionais, incapacidades para manter as habilidades necessárias ao autocuidado, vida independente e autônoma acarretaram maior dependência do cuidador, impactos no funcionamento familiar, dificuldades para gerenciar o cuidado, assim como a busca e o acesso aos serviços especializados. ${ }^{(31,32)}$

A realização de radioterapia e quimioterapia concomitantes associou-se às reaçóes emocionais, maiores implicaçóes na vida pessoal e dificuldades financeiras para o cuidador, em virtude da necessidade maior de locomoção para realização das sessões terapêuticas e pela intensificação de reaçôes adversas, constantemente associada à dor, lesóes de pele, náuseas e vômitos. ${ }^{(29)}$

Além disso, a presença de comorbidades, fatores de risco e hábitos de vida como diabetes, hipertensão, metástase, etilismo e sedentarismo refletiram no maior nível de sobrecarga em diferentes dimensóes do QASCI. O nível de sobrecarga do cuidador de familiar com câncer pode variar em decorrência do estágio da doença e condiçóes clínicas associadas, uma vez que essas características podem contribuir para o aumento do grau de dependência, requerendo maior tempo, dedicação e cuidados específicos. ${ }^{(33)}$
Assim, espera-se colaborar para a produçáo do conhecimento acerca dos indicadores de sobrecarga, fatores associados, repercussões e manifestações clínicas advindas do cuidado, bem como conhecer os determinantes relacionados à saúde do cuidador e subsidiar estratégias de suporte e políticas públicas de saúde que abordem a integração dos serviços e a assistência domiciliar com qualidade, efetividade e segurança.

A limitação do estudo está relacionada à escassez de evidências nacionais e internacionais para comparação dos resultados e ao delineamento utilizado, uma vez que o método transversal não permite estabelecer relaçóes de causa e efeito. Dessa forma, sugerem-se novas investigaçóes voltadas para determinar os preditores de sobrecarga, assim como para estabelecer estratégias de ação que busquem humanizar e qualificar o cuidado, além de minimizar impactos mediante inclusão do cuidador no plano terapêutico.

\section{Conclusão}

O cuidado informal destinado aos pacientes em tratamento radioterápico mostrou-se como fenômeno complexo e foi capaz de gerar moderada sobrecarga física, emocional e social nos cuidadores. As implicaçóes na vida pessoal e os comprometimentos emocionais representaram os principais indicadores de sobrecarga, sendo determinantes para elevação dos escores globais. Aspectos relacionados tanto à demanda de cuidados, quanto às características clínicas e terapêuticas dos pacientes, como o tempo destinado ao cuidado, grau de parentesco, comorbidades e sintomas físicos apresentaram relaçóes positivas, representando os principais fatores associados ao nível de sobrecarga referida pelos cuidadores.

\section{Colaborações}

Pedrosa AO, Rocha DM, e Oliveira AC contribuíram com a concepçáo do projeto, análise e interpretação dos dados e redaçáo do artigo. Santos AMR, Figueiredo MLF, Tolstenko LN contribuíram para 
revisão crítica do conteúdo intelectual e aprovação final da versão a ser publicada.

\section{Referências}

1. Nascimento MI, Silva GA. Waiting time for radiotherapy in women with cervical cancer. Rev Saude Publica. 2015;49(92):1-8.

2. Paula JM, Sawada NO. Health-related quality of life of cancer patients undergoing radiotherapy. Rev Rene. 2015;16(1):106-13.

3. Freire ME, Costa SF, Lima RA, Sawada NO. Health-related quality of life of patients with cancer in palliative care. Texto Contexto Enferm. 2018;27(2):1-13.

4. Bianchin MA, Silva RD, Fuzetto LA, Salvagno V. Sobrecarga e depressão em cuidadores de pacientes oncológicos em tratamento quimioterápico. Arq Ciênc Saúde. 2015;22(3):96-100.

5. Soares ML, Trezza MC, Oliveira SM, Melo GC, Lima KR, Leite JL. The healing cost: comfort and discomfort experiences of women undergoing brachytherapy. Esc Anna Nery. 2016;20(2):317-23.

6. Sousa ÁF, Queiroz AA, Oliveira LB, Moura LK, Andrade D, Watanabe E, et al. Deaths among the elderly with ICU infections. Rev Bras Enferm. 2017;70(4):733-9.

7. Heckel L, Fennell KM, Reynolds J, Boltong A, Botti M, Osborne RH, et al. Efficacy of a telephone outcall program to reduce caregiver burden among caregivers of cancer patients [PROTECT]: a randomised controlled trial. BMC Cancer. 2018;18(1):1-13.

8. Mirsoleymani SR, Rohani C, Matbouei M, Nasiri M, Vasli P. Predictors of caregiver burden in Iranian family caregivers of cancer patients. J Educ Health Promot. 2017;6(1):91.

9. Wittenberg E, Borneman T, Koczywas M, Del Ferraro C, Ferrell B. cancer communication and family caregiver quality of life. Behav Sci (Basel). 2017;7(1):1-12.

10. Monteiro EA, Mazin SC, Dantas RA. The Informal Caregiver Burden Assessment Questionnaire: validation for Brazil. Rev Bras Enferm. 2015;68(3):364-70.

11. Instituto Nacional do Câncer José Alencar Gomes da Silva (INCA). Incidência de câncer no Brasil. Rio de Janeiro: INCA; 2017.

12. Martins T, Ribeiro JP, Garret C. Questionário de avaliação da sobrecarga do cuidador Informal (QASCI): reavaliação das propriedades psicométricas. Rev Ref. 2004;(11):17-31.

13. Pestana MH, Gageiro JN. Análise de dados para ciências sociais: a complementariedade do SPSS. 3rd ed. Lisboa: Sílabo; 2003.

14. Bond SM, Hawkins DK, Murphy BA. Caregiver-reported neuropsychiatric symptoms in patients undergoing treatment for head and neck cancer: a pilot study. Cancer Nurs. 2014;37(3):227-35.

15. Govina 0, Kotronoulas G, Mystakidou K, Katsaragakis S, Vlachou E, Patiraki E. Effects of patient and personal demographic, clinical and psychosocial characteristics on the burden of family members caring for patients with advanced cancer in Greece. Eur J Oncol Nurs. 2015;19(1):81-8.

16. Johansen S, Cvancarova M, Ruland C. The effect of cancer patients' and their family caregivers' physical and emotional symptoms on caregiver burden. Cancer Nurs. 2018;41(2):91-99

17. Anjos C, Espírito Santo FH, Carvalho EM. 0 câncer infantil no âmbito familiar: revisão integrativa. Rev Min Enf. 2015;19(1):227-33.
18. Ringer T, Hazzan AA, Agarwal A, Mutsaers A, Papaioannou A. Relationship between family caregiver burden and physical frailty in older adults without dementia: a systematic review. Syst Rev. 2017 Mar 14;6(1):1-16.

19. Stenberg U, Cvancarova M, Ekstedt M, Olsson M, Ruland C. Family caregivers of cancer patients: perceived burden and symptoms during the early phases of cancer treatment. Soc Work Health Care. 2014;53(3):289-309.

20. Nightingale CL, Curbow BA, Wingard JR, Pereira DB, Carnaby GD. Burden, quality of life, and social support in caregivers of patients undergoing radiotherapy for head and neck cancer: A pilot study. Chronic IIIn. 2016;12(3):236-45.

21. Dean LT, Moss SL, Ransome Y, Frasso-Jaramillo L, Zhang Y, Visvanathan $\mathrm{K}$, et al. "It still affects our economic situation": long-term economic burden of breast cancer and lymphedema. Support Care Cancer. 2019;27(5):1697-708.

22. Srinivasan A, Tiwari K, Scott JX, Ramachandran P, Ramakrishnan M. Impact of cancer support groups on childhood cancer treatment and abandonment in a private pediatric oncology centre. Indian J Palliat Care. 2015 Jan;21(1):68-71.

23. Shahi V, Lapid MI, Kung S, Atherton PJ, Sloan JA, Clark MM, et al. Do age and quality of life of patients with cancer influence quality of life of the caregiver? J Geriatr Oncol. 2014;5(3):331-6.

24. Jesus IT, Orlandi AA, Zazzetta MS. Burden, profile and care: caregivers of socially vulnerable elderly persons. Rev Bras Geriatr Gerontol. 2018;21(2):194-204.

25. Lopez V, Copp G, Molassiotis A. Male caregivers of patients with breast and gynecologic cancer: experiences from caring for their spouses and partners. Cancer Nurs. 2012;35(6):402-10.

26. Souza CP, Silva AG, Quirino AC, Neves MS, Moreira LR. Profiles of dependent hospitalized patients and their family caregivers: knowledge and preparation for domiciliary care practices. Rev Min Enferm. 2014;18(1):173-80.

27. Delalibera M, Barbosa A, Leal I. Circunstâncias e consequências do cuidar: caracterização do cuidador familiar em cuidados paliativos. Cien Saude Colet. 2018;23(4):1105-17.

28. Santos-Orlandi AA, Brito TR, Ottaviani AC, Rossetti ES, Zazzetta MS, Gratão AC, et al. Profile of older adults caring for other older adults in contexts of high social vulnerability. Esc Anna Nery. 2017;21(1):1-8.

29. Bray FN, Simmons BJ, Wolfson AH, Nouri K. Acute and chronic cutaneous reactions to ionizing radiation therapy. Dermatol Ther (Heidelb). 2016;6(2):185-206.

30. Guedes AC, Pereira MG. Burden, coping, physical symptoms and psychological morbidity in caregivers of functionally dependent family members. Rev Lat Am Enfermagem. 2013;21(4):935-40.

31. Rodrigues JS, Oliveira SC, Ferreira NM. Morbidade e perfil de cuidadores familiares de idosos com câncer: um desafio para a saúde pública. Rev Cienc Méd. 2013;22(3):137-45.

32. Souza LR, Hanus JS, Libera LB, Silva VM, Mangilli EM, Simões PW, et al. Sobrecarga no cuidado, estresse e impacto na qualidade de vida de cuidadores domiciliares assistidos na atenção básica. Cad Saude Colet. 2015;23(2):140-9.

33. Borges EL. Franceschini1 J, Costa LH, Fernandes AL, Jamnik S, Santoro IL. Sobrecarga do cuidador familiar: a sobrecarga de cuidar de pacientes com câncer de pulmão, de acordo com o estágio do câncer e a qualidade de vida do paciente. J Bras Pneumol. 2017;43(1):18-23. 